\title{
EL MAGISTERIO DE LOS PAPAS EN LOS MEN- SAJES ANUALES PARA LA JORNADA MUNDIAL DE ORACIÓN POR LAS VOCACIONES
}

DOI: https://doi.org/10.52039/seminarios.v58i203.323

BRENDAN LEAHY*

INTRODUCCIÓN

Los Mensajes para la Jornada Mundial de Oración por las Vocaciones, fueron creados con un triple fin, según dice en su mensaje de 1970 el papa Pablo VI: reflexionar acerca de la múltiple realidad de las vocaciones en la Iglesia, comprometer a todos a colaborar y, sobre todo, pedir al Dueño de la mies que envíe trabajadores a su Iglesia. De cualquier modo, en el transcurso de los 48 mensajes, los papas Pablo VI, el Beato Juan Pablo II y Benedicto XVI nos han ofrecido más, mucho más.

En su introducción a Messaggi per le vocazioni, el obispo Giuseppe Pittau describe los mensajes de la Jornada Mundial de Oración por las Vocaciones «como una pequeña enciclopedia de la teología y (cuidado) pastoral de las vocaciones» ${ }^{1}$. No sólo eso, sino que con la lectura y meditación de estos mensajes, no podemos dejar de lado el carácter excepcional de los Papas que nos los han propuesto. La vida de cada uno de ellos es, por sí misma, un poderoso mensaje en el tema vocacional.

En esta pequeña ponencia en la que presento el Magisterio de los pontífices en los mensajes anuales para la Jornada Mundial de Oración por las Vocaciones, no puedo hacer plena justicia a la riqueza enciclopédica de la enseñanza y observación, sabiduría espiritual y análisis, ejemplos y recomendaciones contenidos en los 48 mensajes. Para realizar este trabajo, me he guiado por un comentario también hecho por el obispo Pittau. Él escribe que «los grandes temas del Concilio en la Iglesia» ${ }^{2}$ se encuentran a lo largo de los mensajes de la Jornada Mundial de Oración por las Vocaciones. Sobre esta base, propongo que

* Profesor de Teología Sistemática en St. Patrick's College, Maynooth (Irlanda). Ponencia en el Congreso de la POVS, Roma, 3-7 noviembre de 2011.

1. Cf. Leonardo Sapienza (ed.), Messaggi per la Giornata Mondiale di Preghiera per le Vocazioni, Rogate, Roma 2003, 14-15. Todas las referencias a los mensajes del papa Pablo VI y Juan Pablo II están tomadas de esta colección. El número de parágrafo remite al parágrafo en ese libro. Los mensajes del papa Benedicto XVI están disponibles en internet.

2. Ibid., 15. 
tracemos juntos las distintas vertientes de la enseñanza del Magisterio papal, bajo tres apartados que fueron prominentes en el Concilio Vaticano II (un evento que cada uno de los tres Papas vivió de una manera muy personal y profunda), y que vuelven una y otra vez como claves significativas para la lectura de las dimensiones teológica, pastoral y espiritual de la vocación. Estos tres apartados son: misterio, comunión y misión.

En la Exhortación Apostólica de 1992, Pastores Dabo Vobis, n. 12, el papa Juan Pablo II se refirió a estas tres claves teológicas como una «síntesis» de la enseñanza del Concilio acerca de la Iglesia. En los primeros tres mensajes, de los seis que ha ofrecido hasta la fecha, el papa Benedicto XVI se ha concentrado precisamente en estos tres temas centrales.

Antes de entrar en tema, me gustaría aclarar un punto. El tema de la vocación concierne a todo ser humano y, particularmente, a todo cristiano bautizado. En última instancia, cada persona encuentra su verdadera identidad en la «entrega sincera de sí mismo», como Gaudium et Spes, 24, lo dice; ésa es la parte central de toda vocación. Varios de los mensajes para la Jornada Mundial de Oración por las Vocaciones lo subrayan. El mensaje de 2001, por ejemplo, dice: «cada vida es vocación». El mensaje de 1971 habla de la «vocación común a ser cristianos», dentro de la cual «cada uno de nosotros está llamado a realizar una función particular para la realización del designio de Dios» (Rom 12, 4-7; 1 Cor 12, 4s). Todos los cristianos son llamados a ayudar a otros a descubrir y realizar su propia vocación ${ }^{3}$. El papa Juan Pablo II escribe: «El descubrimiento de que cada hombre y mujer tiene su lugar en el corazón de Dios y en la historia de la humanidad, constituye el punto de partida para una nueva cultura vocacional $»^{4}$.

De cualquier modo, el enfoque de la mayoría de los mensajes para la Jornada Mundial de Oración por las Vocaciones está, principalmente, en las vocaciones al sacerdocio, a la vida consagrada (sean religiosos o religiosas consagrados en órdenes que llevan siglos de existencia o en las nuevas formas de consagración) y al servicio misionero (ya sea laico u ordenado). Como escribe el papa Benedicto XVI: «Dios siempre ha escogido a algunas personas para colaborar de manera más directa con Él en la realización de su plan de salvación $»^{5}$. Los ejemplos son muchos y se hace referencia a ellos en los mensajes: Moisés y Aarón, Pedro y María, Natanael y Pablo, el Cura de Ars y John Henry Newman.

Son estas «vocaciones para colaborar de manera más directa» con Dios las que los mensajes tienen en mente.

Hechas estas aclaraciones, comencemos. El primer punto es la enseñanza de los Papas sobre la vocación en términos de misterio.

5. Mensaje de 2007. 
I. La VOcAción NACE EN EL «MISTERIO» DE DIOS PRESENTE EN NOSOTROS

\section{Oración}

La primera palabra del primer mensaje transmitido por radio en 1964 es «orar». Es la invocación sugerida por el Señor mismo, que se reproduce como un coro a lo largo de todos estos mensajes: «Rogad por tanto al dueño de la mies que envíe obreros a su mies» (Mt 9, 37). Sí, la vocación es una gracia; está enraizada en una «historia de amor» ${ }^{6}$ que no empieza con nosotros, sino en el misterio de Dios que se nos hace presente en Jesucristo. Desde la eternidad, Dios ha pensado en cada uno de nosotros con miras a una palabra específica que quiere anunciar a la humanidad, como se desarrolla en su plan que se centra en Jesucristo. En la Carta a los efesios leemos: «Bendito sea Dios, Padre de nuestro Señor Jesucristo, que desde lo alto del cielo nos ha bendecido en Cristo... Él nos eligió en Cristo antes de la creación del mundo, para que fuéramos su pueblo y nos mantuviéramos sin mancha en su presencia. Movido por el amor... Él nos ha dado a conocer su plan salvífico que había decidido realizar en Cristo, llevando su proyecto salvador a su plenitud al constituir a Cristo en cabeza de todas las cosas, las del cielo y las de la tierra» (Ef 1, 3-4.9-10).

Los Papas nos invitan en varias ocasiones a meditar sobre cómo, durante su vida terrena, Jesús toma la iniciativa llamando a las personas a descubrir su verdadera identidad siguiéndolo. Vemos esto cuando llama, por ejemplo, a los primeros discípulos, Andrés, Pedro, Santiago y Juan. Así pues, debemos pedir de nuevo, en nuestros días, que él siga llamando y que muchos respondan.

Estos Mensajes nos recuerdan que debemos orar con confianza por esta intención. Los Papas nos invitan a vernos no como extranjeros abandonados por un Dios lejano, sino como personas de fe que creen en la promesa de que Jesús, en el Espíritu Santo, «vendrá al final de los tiempos para congregar a las personas de buena voluntad ${ }^{7}$, que cooperarán en la realización de su plan de unir a la humanidad en una sola familia. Cuando Dios hace una promesa, no engaña.

En su oración, la noche antes de morir, tal como se nos presenta en la Oración sacerdotal del cuarto evangelio, vemos cómo el plan de Dios para cada persona está contenido dentro del diálogo divino del amor: "Yo te ruego por ellos... Haz que ellos sean completamente tuyos por medio de la verdad; tu palabra es la verdad. Yo los he enviado como tú me enviaste a mí. Por ellos yo me consagro a ti... Pero no te ruego solamente por ellos, sino también por todos los que creerán en mí gracias a su palabra. Te pido que todos sean uno lo mismo que lo somos tú y yo, Padre. Y que también ellos vivan unidos a nosotros para que el mundo crea que tú me has enviado» (Jn 17, 9.17-21) ${ }^{8}$.

6. Mensaje de 1984, n. 236.

7. Mensaje de 1972, n. 122.

8. Citado en Mensaje de 1969, n. 66. Cf. también Mensaje de 1978, n. 177. 
Por tanto, ante la escasez de vocaciones en algunas partes del mundo hemos de evitar actitudes de desánimo o pesimismo. Juan Pablo II subrayaba la presencia y ayuda de Cristo Resucitado ${ }^{9}$. Este ha prometido: «Os aseguro que si dos de vosotros se ponen de acuerdo en la tierra para pedir cualquier cosa, la obtendrán de mi Padre del cielo» (Mt 18, 19s). Nuestra plegaria, llamada y respuesta (las tres «claves» en el cuidado de las vocaciones, como las llamó Juan Pablo II en su primer mensaje) deben estar presentes en este horizonte de esperanza.

\section{Vida}

Subrayando que la vocación nace en el misterio de Dios que ha venido entre nosotros, los Papas invitan a contemplar la belleza de la vida que ofrece la vocación. Esta nueva vida resultaba tan fascinante para los primeros discípulos que consideraron secundario todo lo demás.

Benedicto XVI admite que el peso de dos milenios de historia dificulta captar la novedad de esta vida, del «misterio fascinante de la adopción divina» que se ha abierto para nosotros tanto colectiva como individualmente en Cristo: «Estamos llamados a vivir como hermanos y hermanas de Jesús, a sentirnos hijos e hijas de un mismo Padre. Un don que altera cualquier idea y proyecto meramente humanos ${ }^{10}$. Necesitamos combatir la tentación «de sentirnos autosuficientes hasta cerrarnos al misterioso plan de Dios sobre nosotros» (2006).

Juan Pablo II recordaba a menudo que la Jornada Mundial de las Vocaciones tiene lugar entre Pascua y Pentecostés, tiempo litúrgico en el que se nos presenta a Cristo Resucitado, el Buen Pastor, que nos llama a una nueva vida. La vocación es, como él dice, una «llamada a la vida: a recibirla y a darla» "11. Eso es lo que descubrieron quienes encontraron a Jesús. En Él encontramos Vida divina («En la Palabra estaba la vida», cf. Jn 1,4) y nuestra vida se unifica en torno a Él y al Reino. Es por eso por lo que, como expresa Pablo VI, «ninguna cosa, ningún placer, ningún amor puede superar la vocación» ${ }^{12}$.

\section{Libertad}

Si bien la vocación nace en el misterio de Dios presente en nosotros, y si bien la vida que se nos ofrece es fascinante, también existe la misteriosa naturaleza de nuestra respuesta a la vocación. La manera ejemplar para responder la hallamos «cuando algunos pescadores de Galilea, habiendo encontrado a Jesús, se dejaron cautivar por su mirada, por su voz, y acogieron su apremiante invitación:

9. Juan Pablo II, Mensaje de 1979, n. 185

10. Mensaje de 2006.

11. Mensaje de 1982, n. 207.

12. Mensaje de 1974, n. 133. 
'Seguidme, os haré pescadores de hombres' (Mc 1, 17; cf. Mt 4, 19)»'13. En este "dejarse cautivar por su mirada», llegamos a un tema habitual en los mensajes: el tema de la libertad. Los Papas nos llevan a contemplar la gran dignidad y la esplendidez de nuestra condición humana. No «tenemos» que decir «sí» a Dios. La dignidad de nuestra libertad cristiana está en que «podemos» decir sí. La libertad es la base esencial de toda vocación ${ }^{14}$.

El Espíritu del Padre y de Jesús, ciertamente, continúa haciendo resonar dentro de cada persona la llamada más íntima, a la que el papa Pablo VI se refiere como "una aventura de amor $»^{15}$. Como afirma el gran misionero, san Pablo: «A cada cual se le concede la manifestación del Espíritu para el bien de todos... Todo esto lo hace el mismo y único Espíritu, que reparte a cada uno sus dones como él quiere» (1 Cor 12, 7-11).

Son muchas las dificultades que la gente afronta para responder libremente a la llamada. Los Papas son perfectamente conscientes de este problema y mencionan algunas de ellas: el mundo de lo religioso no ejerce la atracción que antes ejerció. En cierto sentido, este es incomprensible para la psicología de los jóvenes ${ }^{16}$. Otra es la cuestión de la Iglesia misma, en su permanente contradicción entre el ideal y la realidad. Desafortunadamente, los escándalos en años recientes han agrandado este problema. También existe una mentalidad generalizada que no favorece el compromiso. Hoy más que en épocas pasadas se necesita un gran esfuerzo personal para ir contra corriente ${ }^{17}$.

Entre las causas de la crisis vocacional los Papas enumeran la crisis de fe y la crisis de amor. Toda vocación nace de la fe, vive en la fe y persevera con fe. Después de todo, «nadie, de hecho, sigue a un extraño; nadie ofrece su vida por un desconocido» ${ }^{18}$. Si la educación en la fe es necesaria, también se necesita la educación en el amor. Toda vocación es un acto de amor en respuesta a Aquel que pregunta: «¿Me amas?... ¿me amas más que éstos [sic]?» (Jn 21, 15.17). Sin conocer la lógica de la fe que trabaja a través del amor, sin una formación espiritual, en la inmensurable medida del amor, los jóvenes se preguntan: «¿Vale la pena?». Al respecto, Pablo VI afirma: «¿No existe más bien crisis de amor, antes que crisis de vocaciones?» ${ }^{19}$. Los jóvenes tienen que ser ayudados para comprobar que los valores positivos del amor humano, riqueza, éxito profesional, placer, prosperidad, poder -en sí mismas cosas buenas-, no son cosas esenciales; y por lo tanto ser ayudados a correr el riesgo de abandonarse a la llamada del más alto amor de todos, Dios, y en Él servir a Jesucristo en su prójimo.

13. Mensaje de 2007.

14. Mensaje de 1968, n. 52.

15. Mensaje de 1967, n. 36.

16. Mensaje de 1968, n. 54.

17. Mensaje de 1974, n. 144.

18. Mensaje de 1977, n. 170.

19. Mensaje de 1977, n. 173. 
Condiciones favorables necesarias para escuchar la Voz

La vocación nace en el misterio y obtiene respuesta en libertad. Ciertamente, en la Iglesia siguen existiendo problemas, cuestiones preocupantes, especialmente en ciertas regiones. Ante ello el papa Pablo VI en su mensaje de 1970 reafirma que la causa de la crisis debería ser buscada en nosotros mismos, más que en los jóvenes. Ante la crisis del consumismo y la crisis de ideales, muchos jóvenes están buscando un estilo de vida auténtico. Esto es terreno fértil para abrir el tema de la vocación. Los Papas nos exhortan a una nueva «creatividad en el amor» y a una nueva imaginación que podríamos utilizar para ayudar a los jóvenes en esta búsqueda, haciendo un uso inteligente de los modernos medios de comunicación social, invitándoles a abrir sus corazones a Cristo.

Los mensajes señalan la necesidad que tiene la persona de ser ayudada para afinar su «sentido espiritual» (escuchar la voz, ver la belleza, desarrollar el gusto de ayudar a otros y servir a la Iglesia ${ }^{20}$ ) con la llamada y belleza de la vocación. Para que eso suceda, ciertamente necesitamos indicar a los jóvenes los medios clásicos que ayudan a fomentar una espiritualidad que facilite el descubrimiento de la vocación: escucha de la Palabra de Dios, participación en los sacramentos -especialmente la Eucaristía y la reconciliación-, oración personal y litúrgica, dirección espiritual, amor a la Virgen María y prácticas ascéticas ${ }^{21}$.

En el transcurso de estos mensajes, los Papas también subrayan la necesidad de crear condiciones favorables para que los jóvenes perciban la vocación ${ }^{22}$. Según sus comentarios, dos caminos importantes deberíamos incluir en nuestra planificación (siempre necesaria según el papa Juan Pablo II) del cuidado de las vocaciones: el camino de la comunión y el camino de la misión.

\section{DISCERNIMIENTO SOBRE EL CAMINO DE LA COMUNIÓN}

\section{Renovando la estructura eclesial}

La Iglesia entera está llamada a crear una atmósfera donde las vocaciones puedan surgir y crecer ${ }^{23}$. Ya en 1972, el papa Pablo VI señaló que el camino de la comunión es importante hoy, ya que una sensibilidad comunitaria está muy viva en nuestro mundo. Juan Pablo II habla de rehacer el entramado cristiano de las comunidades de la Iglesia a la luz de una eclesiología de comunión ${ }^{24}$.

Y eso significa renovar la vida de comunión dentro de la misma Iglesia en todos los niveles: desde la familia -a la cual Juan Crisóstomo llamó «Iglesia do-

20. Mensaje de 1981, nn. 203 y 204.

21. Cf. este resumen en Mensaje de 1990, n. 286.

22. Cf., por ejemplo, Mensaje de 1970, n. 81.

23. Mensaje de 1975, n. 153.

24. Mensaje de 1996, n. 330, citando Christifideles laici, 34. 
méstica», y a la que en el mensaje de 1994 se le llama «primer seminario» y en el mensaje de 1998, «escuela permanente de la civilización del amor»-, hasta la «Iglesia local»; desde la «comunidad parroquial» hasta el «colegio». Partiendo de que «la Iglesia nació para vivir y para dar la vida» ${ }^{25}$, todas las expresiones de la vida de la Iglesia deben asegurarse de que están generando la vida de comunión que Jesús vino a dar en abundancia ${ }^{26}$. Esa vida de comunión se caracteriza por la dinámica de «recibir-dar», que no es sino participar en la propia vida de Dios ${ }^{27}$. En el mensaje de 2003, el papa Juan Pablo II afirmó: «Cuando las relaciones interpersonales son inspiradas en el servicio recíproco, se crea un mundo nuevo y en él se desarrolla una auténtica cultura vocacional». Esto es importante en la creación de «una Iglesia para los jóvenes» ${ }^{28}$. Por lo tanto, un primer paso esencial en la promoción vocacional es garantizar que la vocación cristiana, en sí misma -basada en el bautismo, la confirmación y la Eucaristía-, se mantenga viva; lo que sería «perfectamente cristiano» (cf. Mt 5, 48) ${ }^{29}$. Ello implica un continuo redescubrimiento del Evangelio: «Una comunidad que no vive generosamente conforme al Evangelio, no puede ser más que una comunidad pobre de vocaciones» ${ }^{30}$.

Como dice el mensaje de 1982, sólo la vida genera vida. Todos estamos llamados a ser testigos del gozo y plenitud que acompaña a la vida de comunión con Cristo y con los demás, a la luz del Evangelio. Sobre todo, aquellos que ya viven una vocación especial -sacerdote, consagrado o misionero- están llamados a dejarse atraer y conquistar por su vocación, viviéndola auténticamente en comunión, y así fascinar y atraer a otros.

\section{Las dimensiones subjetiva y objetiva de la vocación}

Hay un punto importante que varios mensajes aclaran al explicar por qué la vida en comunión con otros facilita a las personas distinguir la «voz» de Dios. Se nos recuerda que cada vocación incluye dos aspectos ${ }^{31}$. Por un lado, está la expresión «interior» subjetiva de la voz dentro de nosotros, ésa del Espíritu Santo; la «voz silenciosa» que se percibe en lo profundo de cada persona. Puede haber, por supuesto, «destellos de iluminación» en los que el individuo percibe la llamada. Pero después hay, como hubo, la necesidad de un altavoz que ayude a que esa voz sea escuchada en los instrumentos «exteriores» de los medios humanos, sociales y concretos de la Iglesia, tales como la Palabra

25. Mensaje de 1982, n. 209.

26. El título del Mensaje de 1982 es «La vida genera vida».

27. Mensaje de 1982, n. 213.

28. Mensaje de 1995, n. 323.

29. Mensaje de 1970, n. 85. Cf. también 1983, n. 222.

30. Mensaje de 1970, n. 86. Cf. también Mensaje de 1996, n. 344.

31. Cf. Pablo VI, Audiencia General, 5 de mayo de 1965, 17. 
de Dios proclamada, la jerarquía, la llamada explícita, el diálogo personal y la animación por parte de la familia ${ }^{32}$.

Es imprescindible, en una cultura que tan frecuentemente exalta lo subjetivo, situar ante la dimensión objetiva, exterior de la vocación. La vocación no es sólo un sentimiento interior. Viene a nosotros desde fuera. Es algo que es provocado, motivado, discernido y confirmado en interacción con otros. Si la vida en comunión está verdaderamente viva, ésta genera una atmósfera en la que las vocaciones se tornan claras, las dificultades se superan y se hace más fácil decir «sí».

Como afirma el papa Benedicto XVI: «Esa intensa comunión favorece el florecimiento de generosas vocaciones para el servicio de la Iglesia... Para promover vocaciones es por tanto importante una pastoral atenta al misterio de la Iglesia-comunión, porque quien vive en una comunidad eclesial concorde, corresponsable, atenta, aprende ciertamente con más facilidad a discernir la llamada del Señor» ${ }^{33}$.

\section{Siendo y diciendo. El diálogo pastoral}

Considerando la contribución de la vida en comunión, podemos notar una doble tarea en la promoción vocacional: «ser» y «decir».

Todos nosotros estamos llamados a «ser» o «atestiguar» a través de nuestras vidas, la nueva vida de comunión que se devela en Jesucristo. Si los jóvenes no ven esa vida, nunca escucharán la llamada. Como dice el mensaje de 2010: «El testimonio suscita vocaciones». Nosotros mismos constantemente necesitamos ser evangelizados y evangelizar ${ }^{34}$. Desde la eclesiología de comunión, eso supone también una conversión ministerial a la colaboración entre los mismos pastores y entre los pastores y los fieles laicos.

Pero también debemos «hablar» de la vida en comunión. «Ser una comunidad que llama», escribe Juan Pablo II en su mensaje del año 1986, indicando la necesidad de pasar de una pastoral «de la espera» a una «pastoral de la propuesta». En los mensajes, las palabras del gran misionero Pablo aparecen citadas con frecuencia: "¿Y cómo van a creer en él, si no les ha sido anunciado?» (Rom 10, 14). Un rector de un seminario alemán en cierta ocasión dijo: «¡Hay muchos Samueles, pero pocos Elís!». En los Mensajes se nos recuerda que es importante hablar. Tener el valor de tocar el tema de la vocación con una persona es signo de estima hacia esa persona, y puede ser un verdadero momento de gracia en sus vidas.

32. Beda el Venerable es citado en el Mensaje de 1974, n. 138: «El Señor que llamó a Leví exteriormente con la palabra, lo provocó interiormente con una inspiración divina para que inmediatamente siguiera a Aquel que lo llamó».

33. Mensaje de 2007. Cf. también Mensaje de 1997, 345 y 346.

34. En el Mensaje de 1976, n. 158, Pablo VI escribe: «Ahora depende de nosotros... recibir su palabra y darla; para vivirla y dar testimonio de ella; para ser evangelizados y evangelizar». 
El «diálogo pastoral», como el papa Pablo VI indica, es un instrumento importante para el cuidado de las vocaciones. Ésta es la lógica del «cor ad cor loquitun», proveniente del «sentido de la caridad» que recuerda que cada persona es única ${ }^{35}$. Debemos desarrollar más este coloquio personal con los jóvenes, ayudándolos a tomar buenas decisiones. En su último mensaje, el papa Juan Pablo II comentó que hay una necesidad urgente de implementar un amplio plan de promoción vocacional basado en el contacto personal. Los seminaristas también deben ser «los primeros animadores de vocaciones entre los jóvenes de su edad, irradiando los ideales que mueven su existencia» ${ }^{36}$.

Algunos de los pasajes más bellos de estos mensajes son aquellos en los que los Papas hablan directamente a los jóvenes ${ }^{37}$. Valdría la pena recopilar y publicar una selección de estos grandiosos textos personales y directos.

\section{RECORRIENDO EL CAMINO DE LA MISIÓN}

\section{Formar y fomentar la generosidad de los jóvenes}

El segundo camino importante en el cuidado de las vocaciones que se observa en estos mensajes es el de la misión. La vida que nace en Dios, vivida y comunicada dentro de la comunidad, está destinada a alcanzar a todos. Los mensajes sugieren que fomentemos las vocaciones con una verdadera educación en la fe que haga a los jóvenes mirar hacia el exterior y no sólo dentro de ellos mismos, atreviéndose a «afrontar riesgos» por el Evangelio y ser constructores de un nuevo mundo a la luz de la fe (2008).

En el mensaje de 2003 leemos que «no obstante cierto impulso contrario también presente en la mentalidad actual, se da en el corazón de muchos jóvenes una natural disposición a abrirse al otro, de forma especial al más necesitado. Todo ello les hace generosos, capaces de empatía, dispuestos a olvidarse de sí mismos para anteponer al otro a sus propios intereses ${ }^{38}$. Los jóvenes no quieren palabras, sino hechos. Quieren construir un mundo nuevo. Necesitamos aprovechar este dinamismo juvenil y fomentar el compromiso misionero.

Las familias pueden crear tal atmósfera y «comunicar el gusto de ayudar al prójimo y de servir a la Iglesia, cultivar las buenas disposiciones para acoger y seguir la voluntad del Señor ${ }^{39}$. El colegio católico, además de dar razones para decir «sí» a una vocación especial, también «prestará una ayuda valiosa (...)

35. Cf. Audiencia General, 5 de mayo de 1965.

36. Mensaje de 1984, n. 233.

37. En su Mensaje de 1995, el papa Juan Pablo II señala que los jóvenes corren el riesgo de ser privados de una auténtico crecimiento porque no encuentran a nadie a quién hacer la pregunta que hizo el joven rico: ¿Qué debo hacer para heredar la vida eterna?

38. Mensaje de 2003, n. 392.

39. Mensaje de 1981, n. 204. 
favoreciendo experiencias y creando un ambiente de fe, de generosidad y de servicio que puedan librar a los jóvenes de aquellas condiciones que hacen aparecer 'no apetecible' o imposible la respuesta a la llamada de Cristo» ${ }^{40}$.

Desde el punto de vista sociológico-religioso, es bueno ayudar a los jóvenes a escuchar los gritos de la humanidad y a encontrar la manera de responder. Es esencial que escuchen el llanto del pobre y del que sufre, que esperan que alguien se interese por ellos.

Pablo VI habla de una "sinfonía de las vocaciones» que es escuchada en estos llantos ${ }^{41}$. Los valores de la solidaridad, fraternidad, sacralidad de la vida, se vuelven más claros cuando tendemos la mano a los necesitados. En este sentido, el papa Juan Pablo II escribió en su mensaje de 2003 que «la diakonía es un verdadero itinerario de pastoral vocacional», ayudando a las personas a comprender mejor su vocación ${ }^{42}$. Ellos comienzan a ver que «la vida debe ser consagrada a algo grande $»^{43}$ si es para responder adecuadamente.

\section{Ayudar a los jóvenes a leer "los signos del Espíritu»}

Involucrar a los jóvenes en la misión apostólica es bueno, pero no suficiente. ¡Necesitamos personas que se comprometan en la vida misionera apostólica de forma permanente!

Como apuntó Pablo VI, los jóvenes deben pasar del plano psicológico al religioso y leer los misteriosos «signos del Espíritu» que los llaman a la santidad ${ }^{44}$. Pero no pueden hacer esto por ellos mismos. Para esto se necesita una «lectura carismática» de la realidad ${ }^{45}$. Necesitan superar la brecha entre fe y vida, fe y cultura. Los jóvenes deben ser ayudados a ver que «el mundo no puede ser transformado ni ofrecido a Dios sin el espíritu de las bienaventuranzas ${ }^{46}$. Ellos también deben estar atentos a la gran llamada misionera «que siempre necesita donaciones radicales y totales, impulsos nuevos y valientes $»^{47}$.

\section{Llamada radical al servicio como voluntarios de la Cruz}

Los Papas recuerdan que se necesita ofrecer a los jóvenes la experiencia de ser «voluntarios de la Cruz y de la Gloria de Cristo» ${ }^{48}$. El Jesús que atrae es el «Jesús siervo» que toma la condición de esclavo (Flp 2, 7-8) y que entregó

40. Mensaje de 1989, n. 277.

41. Mensaje de 1974, n. 135. Cf. también Juan Pablo II, Mensaje de 1985, n. 242.

42. New Vocations for a New Europe, 23c citado en el Mensaje de 1003.

43. Mensaje de 1971, n. 101.

44. Mensaje de 1974, n. 136 y Mensaje de 1971, n. 106.

45. Mensaje de 1974, n. 136.

46. Mensaje de 1969, n. 63 citando Lumen Gentium, 31.

47. Mensaje de 2008 citando la encíclica misionera de Juan Pablo II, Redemptoris Missio, 66.

48. Audiencia General, 5 de mayo de 1965, n. 19. 
su vida por los otros. El Buen Pastor dijo: «Yo doy mi vida... la doy por mi propia voluntad» (Jn 10, 17s). Y en la Carta a los efesios leemos que Él ofreció su vida al servicio de la Iglesia: «Cristo amó a su Iglesia y se entregó a sí mismo por ella» (Ef 5,25$)$. Es en su seguimiento y en la llamada al servicio donde el joven descubre su verdadera identidad en una especie de muerte y resurrección, propia de toda llamada a la misión en el servicio generoso a Dios y al prójimo.

Finalmente, es aprendiendo a amar al Cristo Crucificado como las personas descubren una lógica que las lleva en direcciones que nunca habrían imaginado: «Te aseguro que cuando eras más joven, tú mismo te ceñías e ibas adonde querías; pero cuando seas viejo extenderás los brazos y será otro quien te ciña y te conducirá adonde no quieras ir» (Jn 21, 18). Respondiendo «sí» a esta lógica, escondida en Cristo, se encuentra la verdadera libertad y felicidad.

\section{CONCLUSIÓN}

En esta ponencia he intentado recopilar las líneas principales de los mensajes de los Papas en torno a los tres temas centrales de misterio, comunión y misión. Estos no pueden ser tomados como dimensiones separadas en el cuidado pastoral de las vocaciones; se da una mutua interacción entre ellos.

No hay duda de que los lectores de estos mensajes percibirán la preocupación de los Papas: hay una necesidad urgente de vocaciones al ministerio ordenado, vida consagrada y misionera. El cuidado pastoral de las vocaciones es uno de los temas más importantes que enfrenta la Iglesia. Los sacerdotes son necesarios porque la misma «constitución» de la Iglesia demanda que haya tales vocaciones en su misma estructura de Iglesia dispuesta por Cristo («Como el Padre me ha enviado, yo también os envío a vosotros» [Jn 20, 21]). Consagrados y consagradas que vivan los consejos evangélicos son necesarios, porque sin ellos la Iglesia se empobrecería. Son necesarias personas que miran hacia la eternidad: El mandato universal a la misión los reclama.

La llamada a la acción de estos mensajes va dirigida a toda la Iglesia, y especialmente a los obispos, presbíteros, personas consagradas y educadores. En su mensaje de 2005 (escrito en agosto de 2004), Juan Pablo II concluye: «Los jóvenes necesitan de Cristo, pero saben también que Cristo quiere contar con ellos». Finalmente, los mensajes animan a cada obispo, presbítero, persona consagrada y misionero a ofrecer su propio testimonio. Muchos están a la espera de escuchar nuestra historia y la fascinación que hemos experimentado al ser llamados. Después de todo, como dijo recientemente Benedicto XVI a los jóvenes en Madrid: "Qué hermoso es saber que Jesús te busca, se fija en ti y con su voz inconfundible te dice: ‘¡Sígueme!'» (cf. Mc 2, 14). 